08,04

\title{
Парамагнитные центры в легированном хромом монокристалле $\mathrm{Na}_{5} \mathrm{AlF}_{2}\left(\mathrm{PO}_{4}\right)_{2}$
}

\author{
(C) В.А. Важенин, А.П. Потапов, А.В. Фокин, М.Ю. Артёмов \\ Уральский федеральный университет, Институт естественных наук и математики, \\ Екатеринбург, Россия \\ E-mail: Vladimir.Vazhenin@urfu.ru
}

Поступила в Редакцию 19 июня 2021 г.

В окончательной редакции 19 июня 2021 г.

Принята к публикации 3 июля 2021 г.

Исследован парамагнитный резонанс монокристаллов $\mathrm{Na}_{5} \mathrm{AlF}_{2}\left(\mathrm{PO}_{4}\right)_{2}$ с примесью хрома. Обнаружены центры $\mathrm{Cr}^{3+}$, заместившие триклинные позиции $\mathrm{Al}^{3+}$. В локальной системе координат спектры этих центров описываются спиновым гамильтонианом ромбической симметрии. Кроме того, наблюдаются центры ванадия и не идентифицированные центры триклинной симметрии.

Ключевые слова: примесные ионы, фосфат фторида алюминия, парамагнитный резонанс.

DOI: 10.21883/FTT.2021.11.51597.150

\section{1. Введение}

В процессе поиска новых суперионных материалов, в которых проводимость обеспечивается переносом ионов натрия [1], авторы работ [2-5] провели исследования кристаллической структуры и ионной проводимости соединений $\mathrm{Na}_{5} \mathrm{AlF}_{2}\left(\mathrm{PO}_{4}\right)_{2}$ и $\mathrm{Na}_{5} \mathrm{GaF}_{2}\left(\mathrm{PO}_{4}\right)_{2}$; в обоих кристаллах был обнаружен структурный переход при $\sim 545 \mathrm{~K}$. Величина ионной проводимости этих соединений $\left(\sim 10^{-7} \mathrm{~S} / \mathrm{cm}\right.$ при $\left.293 \mathrm{~K}\right)$ увеличивается с ростом температуры, достигая значения $\sim 10^{-4} \mathrm{~S} / \mathrm{cm}$ при $600 \mathrm{~K}[4]$.

Сообщение о первом исследовании кристаллической структуры $\mathrm{Na}_{5} \mathrm{AlF}_{2}\left(\mathrm{PO}_{4}\right)_{2}$ появилось в работе [2]. С точки зрения параметров элементарной ячейки и пространственной группы симметрии структура, полученная в [3] для соединения $\mathrm{Na}_{5} \mathrm{AlF}_{2}\left(\mathrm{PO}_{4}\right)_{2}$, согласуется с результатами, описанными в [2]. Однако $z$-координаты атомов в этих двух исследованиях, очень близкие по своим значениям, отличаются знаком. Изменение знака параметра $z$ должно привести к иной ориентации многогранников (тетраэдров $\mathrm{PO}_{4}$ и октаэдров, содержащих ионы алюминия или натрия).

Настоящая работа представляет исследование методом электронного парамагнитного резонанса (ЭПР) монокристалла $\mathrm{Na}_{5} \mathrm{AlF}_{2}\left(\mathrm{PO}_{4}\right)_{2}$, легированного хромом, с целью сравнения ориентаций главных осей парамагнитных центров с элементами полиэдров двух представленных структур [2,3].

\section{2. Образцы и методика измерений}

Монокристаллы $\mathrm{Na}_{5} \mathrm{AlF}_{2}\left(\mathrm{PO}_{4}\right)_{2}$ были выращены в Институте кристаллографии им. А.В. Шубникова РАН методом кристаллизации из раствора-расплава [6] при температуре $\sim 1000 \mathrm{~K}$. Измерения проводились при комнатной температуре на спектрометре ЭПР трехсантиметрового диапазона EMX Plus Bruker. Образцы, ориентированные на рентгеновском дифрактометре, помещались в микроволновый резонатор на держатель, обеспечивающий вращение как в горизонтальной, так и в вертикальной плоскостях.

Пространственная группа $\mathrm{Na}_{5} \mathrm{AlF}_{2}\left(\mathrm{PO}_{4}\right)_{2}$ при комнатной температуре $P \overline{3}\left(C_{3 i}^{1}\right)$, параметры решетки: $a=b=10.483(1) \AA, c=6.607(1) \AA$ (из работы [2]), $a=b=10.468(3) \AA, c=6.599(2) \AA \quad$ (из работы [3]). Структура кристалла содержит шесть независимых позиций ионов натрия, имеющих координационное число шесть и точечные группы симметрии $C_{3 i}$ (2 позиции), $C_{3}(2), C_{i}(1)$ и $C_{1}(1)$ [2-3]. Окружение разных ионов натрия как чисто кислородное, так и смешанное - фторкислородное. Среднее расстояние фосфор-кислород в тетраэдрах $\mathrm{PO}_{4}$ имеет размер, типичный для ортофосфатов [2]. Ионы алюминия в этом кристалле имеют одну позицию с симметрией $\overline{1}\left(C_{i}\right)$ (и окружены четырьмя кислородами и двумя фторами, при этом октаэдр сжат в направлении фтор-фтор [2,3].

\section{3. Экспериментальные результаты}

Образцы $\mathrm{Na}_{5} \mathrm{AlF}_{2}\left(\mathrm{PO}_{4}\right)_{2}$ демонстрируют в $X$-диапазоне интенсивный спектр ЭПР, при этом большинство наблюдаемых сигналов принадлежит (в соответствии с кристаллической структурой) центрам с триклинной симметрией. Об этом говорит тот факт, что при $B \| C_{3}$ $(B-$ индукция магнитного поля, см. рис. 1) почти все сигналы собираются в эквивалентные группы (тройки). Это хорошо видно на рис. 2, представляющем ориентационную зависимость положений ЭПР сигналов, где магнитное поле вращается в произвольной плоскости, содержащей ось $C_{3}$ кристалла. 


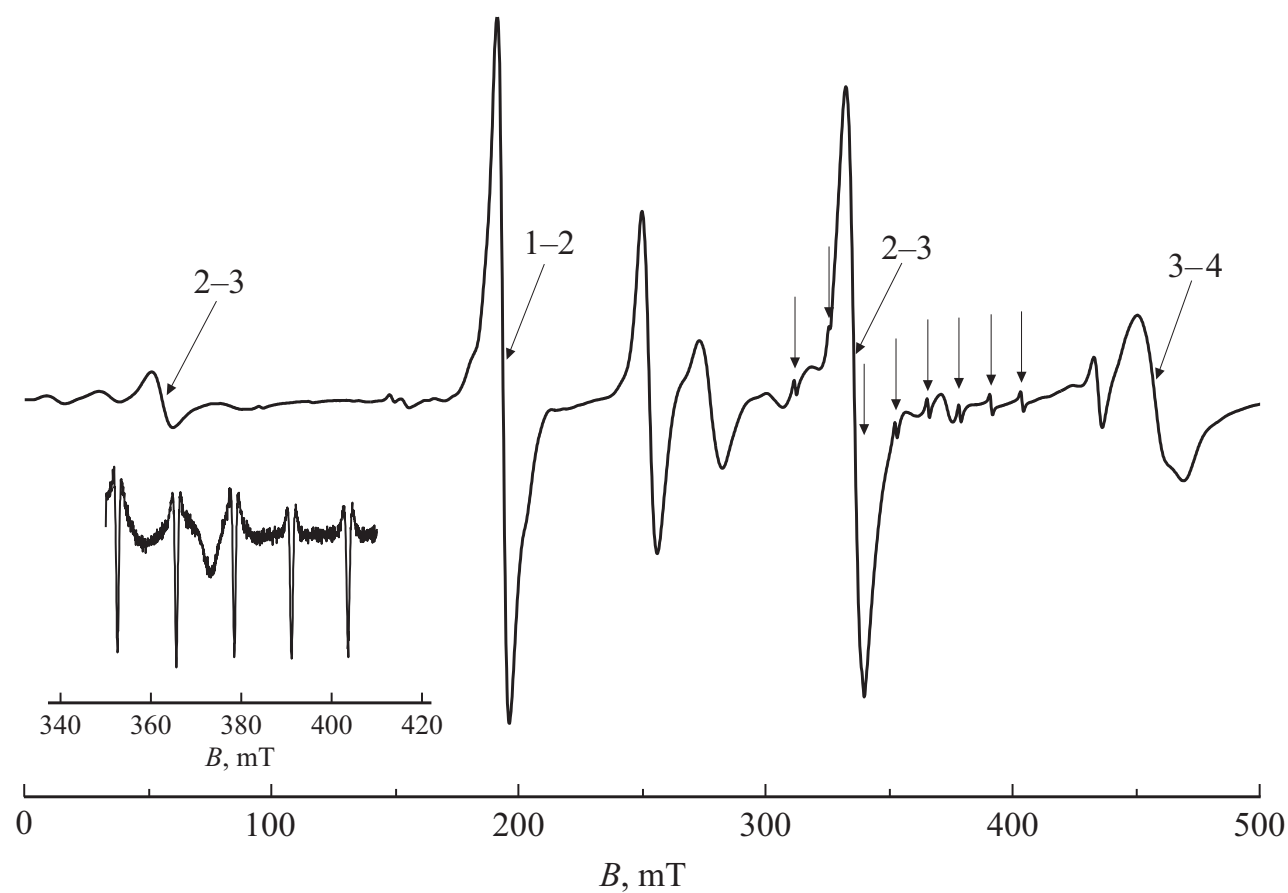

Рис. 1. Спектр ЭПР (производная сигналов поглощения) $\mathrm{Na}_{5} \mathrm{AlF}_{2}\left(\mathrm{PO}_{4}\right)_{2}$ : Сr при $B \| C_{3}$ и $300 \mathrm{~K}$ на частоте $9849 \mathrm{MHz}$. Стрелки с цифрами показывают тип перехода центров $\mathrm{Cr}^{3+}$, уровни энергии нумеруются снизу вверх. Вертикальные стрелки указывают на компоненты сверхтонкой структуры ванадия. На вставке - вторая производная верхних по полю компонент сверхтонкой структуры центров ванадия.

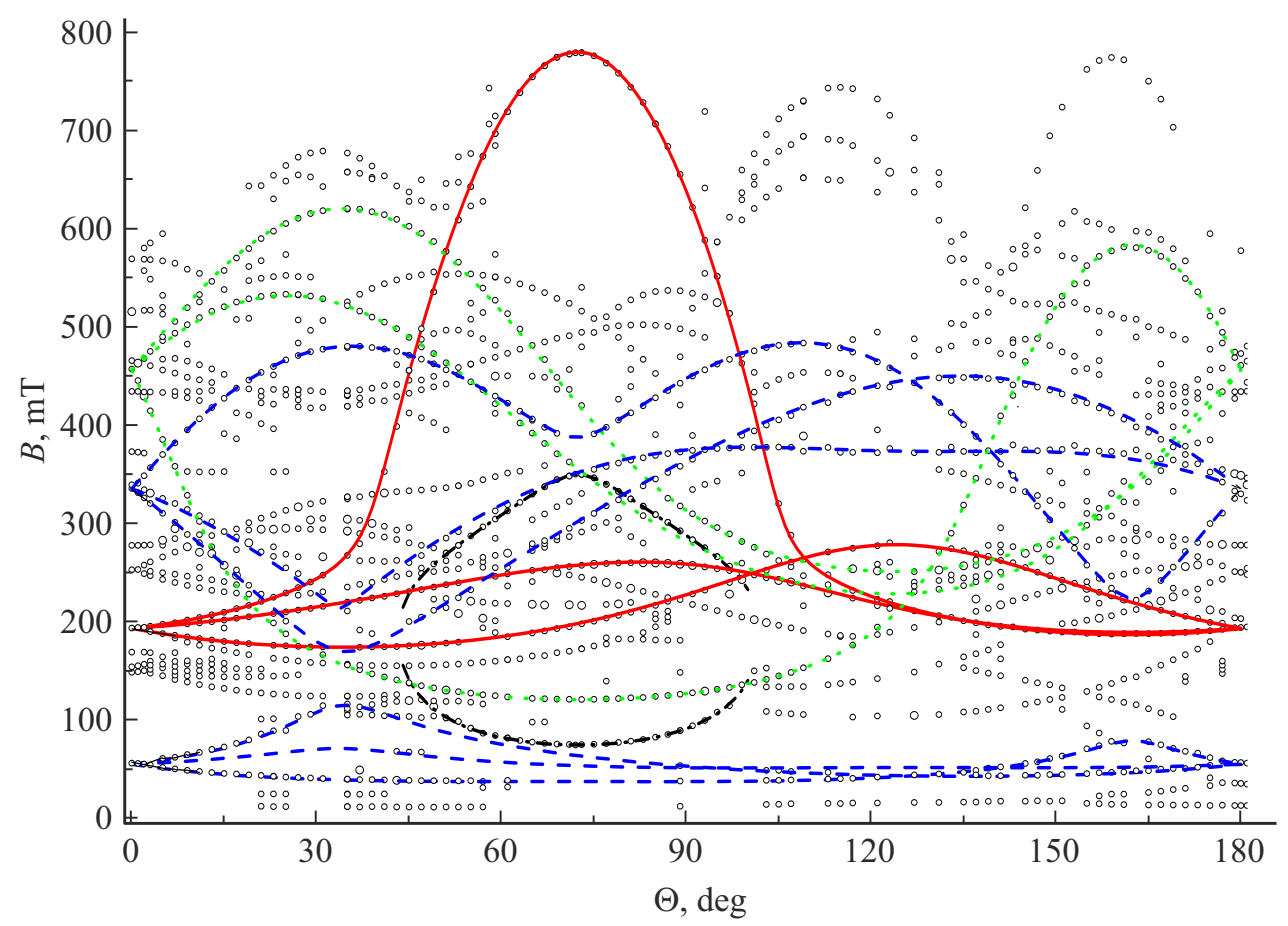

Рис. 2. Ориентационное поведение положений ЭПР-переходов $\mathrm{Na}_{5} \mathrm{AlF}_{2}\left(\mathrm{PO}_{4}\right)_{2}: \mathrm{Cr}$ в произвольной плоскости, содержащей ось $C_{3}$ кристалла $\left(Z \| C_{3}\right)$. Кривые - результат расчета с гамильтонианом (1) и параметрами (2). Красные сплошные кривые переход (1-2), синие штриховые - $(2-3)$, зеленые пунктирные $(3-4)$, черные штрих-пунктирные $-(1-3)$. 


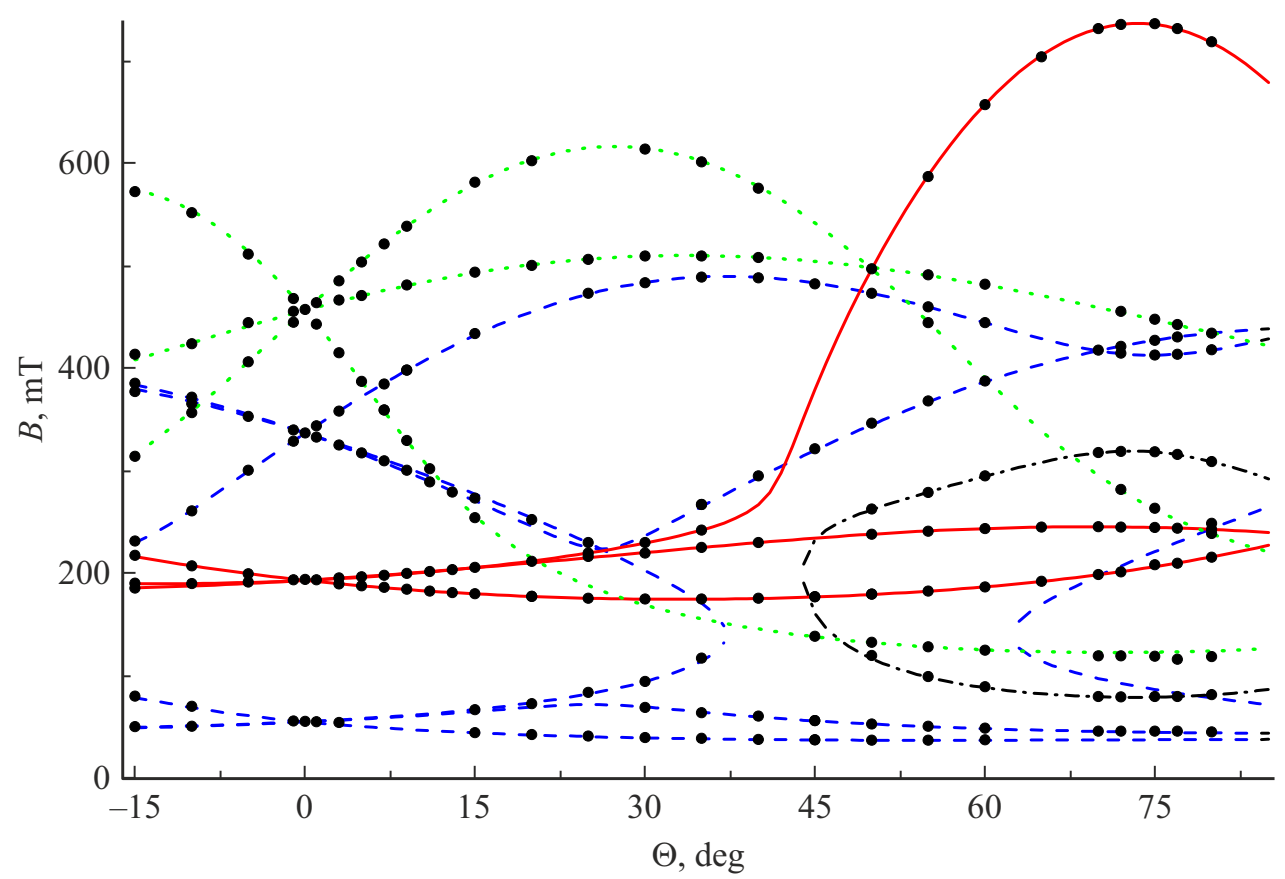

Рис. 3. Ориентационное поведение положений ЭПР-переходов $\mathrm{Na}_{5} \mathrm{AlF}_{2}\left(\mathrm{PO}_{4}\right)_{2}$ : $\mathrm{Cr}$ в плоскости, перпендикулярной оси b, на частоте 9849 MHz. Кривые - результат расчета с гамильтонианом (1) и параметрами (3). Красные сплошные кривые - переход (1-2), синие штриховые - (2-3), зеленые пунктирные (3-4), черные штрих-пунктирные - (1-3).

Анализ угловых зависимостей резонансных положений сигналов на рис. 2 позволил выделить интенсивные переходы трех парамагнитных центров со спином $S=3 / 2$, переходящих друг в друга при повороте на $120^{\circ}$ вокруг $C_{3}$. Учитывая, что кристалл легировался хромом, наблюдаемые центры отнесены к ионам $\mathrm{Cr}^{3+}(S=3 / 2)$, заместившим ионы $\mathrm{Al}^{3+}$ (примесные ионы $\mathrm{Cr}^{3+}$ в структурах корунда, граната, хризоберилла, шпинели, алюмината иттрия, галлата лантана занимают октаэдрически координированные позиции алюминия или галлия). Большая ширина сигналов $\left(\Delta B_{p p} \geq 5 \mathrm{mT}\right)$ не позволяет детектировать сверхтонкую (от ${ }^{53} \mathrm{Cr}$ с ядерным спином $I=3 / 2$ и распространенностью $9.55 \%$ ) и суперсверхтонкую (от $\left.{ }^{19} \mathrm{~F} \mathrm{c} I=1 / 2\right)$ структуры.

Для параметров спинового гамильтониана [7] указанных центров $\mathrm{Cr}^{3+}$

$$
\begin{aligned}
H_{s p}= & \beta(\mathbf{B g S})+1 / 3\left(b_{20} O_{20}+b_{21} O_{21}\right. \\
& \left.+b_{22} O_{22}+c_{21} \Omega_{21}+C_{22} \Omega_{22}\right),
\end{aligned}
$$

где $\mathbf{g}-g$-тензор, $\beta-$ магнетон Бора, $O_{2 m}=O_{2}^{m}$ и $\Omega_{2 m}=\Omega_{2}^{m}-$ спиновые операторы Стивенса [7], $b_{2 m}=b_{2}^{m}$ и $c_{2 m}=c_{2}^{m}-$ параметры тонкой структуры [7], в системе координат $Z \| c$ кристалла получены следующие значения (среднеквадратичное отклонение $53 \mathrm{MHz}$ по 216 экспериментальным положениям переходов, см. рис. 2):

$$
\begin{aligned}
& g=1.973, \quad b_{20}=-2501 \mathrm{MHz}, \quad b_{21}=10704 \mathrm{MHz}, \\
& b_{22}=7500 \mathrm{MHz}, \quad c_{21}=2564 \mathrm{MHz}, \quad c_{22}=-825 \mathrm{MHz} .
\end{aligned}
$$

Измерения при гелиевых температурах не проводились, вследствие чего абсолютные знаки параметров тонкой структуры определены не были. Приведенные в (2) знаки обусловлены первоначальным выбором уровней энергии (как 1-2) для самого высокополевого перехода в ориентационной зависимости (рис. 2) при $\theta=72^{\circ}$. Параметры (2) были определены численными методами с использованием комплексной матрицы энергии 4-го порядка $(2 \cdot S+1)$.

Кривые на рис. 2 представляют результаты расчета угловых зависимостей положений переходов трех центров $\mathrm{Cr}^{3+}$, развернутых вокруг $C_{3}$ на углы $\pm 120^{\circ}$, с гамильтонианом (1) и параметрами (2).

Чтобы связать локальную систему координат центров $\mathrm{Cr}^{3+}$ с псевдоосями искаженного кислородно-фторового октаэдра, содержащего ион алюминия, были проведены измерения ориентационного поведения ЭПР спектра при вращении магнитного поля вокруг кристаллографической оси b (рис. 3). В прямоугольной системе координат, оси которой связаны с осями кристалла $Z \|$ с и $Y \| \mathrm{b}$, для параметров спинового гамильтониана (1) центра $\mathrm{Cr}^{3+}$ при обработке 128 резонансных положений переходов получены следующие величины:

$$
\begin{aligned}
& g=1.973, \quad b_{20}=-2506 \mathrm{MHz}, \quad b_{21}=9914 \mathrm{MHz}, \\
& b_{22}=7120 \mathrm{MHz}, \quad c_{21}=4870 \mathrm{MHz}, \quad c_{22}=2420 \mathrm{MHz}
\end{aligned}
$$

(при среднеквадратичном отклонении $20 \mathrm{MHz}$ ).

Предыдущая плоскость, в которой проводились измерения (рис. 2), отстоит от данной (рис. 3) на $12.5^{\circ}$. 
Следует заметить, что поведение угловых зависимостей (особенно перехода 2-3) на рис. 2, 3 заметно отличается. При изотропном $g$-факторе и отсутствии сверхтонкой структуры главные оси центра можно найти, вращая систему координат для максимизации абсолютного значения параметра $b_{20}$ тензора тонкой структуры. Для центра $\mathrm{Cr}^{3+}$ в локальной (главной) системе координат $(x y z)$, параметр $b_{20}$ имеет максимальное значение, а тензор второго ранга содержит только два параметра

$$
b_{20}=5857 \mathrm{MHz} \text { и } b_{22}=1730 \mathrm{MHz} \text {. }
$$

Таким образом, в пределах погрешности измерений центр $\mathrm{Cr}^{3+}$ можно считать ромбическим. Данная система координат $x y z$ связана с системой $X Y Z$ углами Эйлера $\alpha_{1}=11.13^{\circ}, \beta_{1}=72.31^{\circ}, \gamma_{1}=120.17^{\circ}$, задающими последовательные повороты вокруг осей $z y z$. Системы координат двух других магнитно-неэквивалентных центров получаются дополнительным поворотом на 120 градусов вокруг оси $C_{3}$ кристалла: $\alpha_{2}=131.13^{\circ}$, $\beta_{2}=72.31^{\circ}, \gamma_{2}=120.17^{\circ}$ и $\alpha_{3}=-108.87^{\circ}, \beta_{3}=72.31^{\circ}$, $\gamma_{3}=120.17^{\circ}$.

Как видно на рис. 1-2, в ЭПР спектре $\mathrm{Na}_{5} \mathrm{AlF}_{2}\left(\mathrm{PO}_{4}\right)_{2}: \mathrm{Cr}$ присутствует еще довольно большое количество более слабых, нами не идентифицированных сигналов. Эти сигналы могут быть обусловлены ионами группы железа, сопутствующими примеси хрома. В частности, вблизи $g=2$ (рис. 1) наблюдается октет узких (ширина $\Delta B_{p p} \approx 1 \mathrm{mT}$ ) слабых сигналов протяженностью $92 \mathrm{mT}$. Такая сверхтонкая структура скорее всего обусловлена изотопом иона ванадия ${ }^{51} \mathrm{~V}$, имеющим ядерный спин $I=7 / 2$ и естественную распространенность 99.76\% (спектр ЭПР кобальта с $I=7 / 2$, распространенностью $100 \%$ и $g>2$ при комнатной температуре наблюдается крайне редко).

Поскольку ионы ванадия $\left(\mathrm{V}^{2+}, \mathrm{V}^{3+}, \mathrm{V}^{4+}\right)$ хорошо замещают катионы с октаэдрическим окружением в кристаллах $\mathrm{Al}_{2} \mathrm{O}_{3}, \mathrm{Y}_{3} \mathrm{Al}_{5} \mathrm{O}_{12}, \mathrm{MgO}, \mathrm{CaO}, \mathrm{KMgF}_{3}, \mathrm{KCdF}_{3}$, $\mathrm{NaCl}$ наблюдаемый в $\mathrm{Na}_{5} \mathrm{AlF}_{2}\left(\mathrm{PO}_{4}\right)_{2}$ спектр можно было бы отнести к центрам ванадия, локализованным в низко симметричных позициях $\mathrm{Al}^{3+}$. Тем более, что указанные сигналы при уходе от ориентации $B \| C_{3}$ расщепляются на три компоненты. Парамагнитные центры ванадия в кристаллах $\mathrm{KMgF}_{3}$ и $\mathrm{CaF}_{2}$, имея фторовое окружение [8-10], как правило, демонстрируют суперсверхтонкую структуру, обусловленную взаимодействием с ядерным спином фтора $(I=1 / 2$, распространенность 100\%). Отсутствие каких-либо признаков суперсверхтонкой структуры (см. вставку на рис. 1) при наличии в ближайшем окружении позиции иона $\mathrm{Al}^{3+}$ на расстоянии $1.828 \AA$ двух ионов фтора позволяет допустить иную локализацию ванадия. Например, замещение ионом ванадия позиции натрия с кислородным окружением (точечная симметрия $C_{3 i}$ или $C_{3}$ ) и локальной компенсацией заряда вакансией соседнего натрия.

\section{4. Обсуждение результатов}

На рис. 4.1 приведено положение оси $z$ локальной системы координат относительно трех полиэдров $\mathrm{AlO}_{4} \mathrm{~F}_{2}$ в структуре кристалла $\mathrm{Na}_{5} \mathrm{AlF}_{2}\left(\mathrm{PO}_{4}\right)_{2}$ согласно работе [2]. Хорошо видно, что главная локальная ось близка к квази-тригональным осям трех кислородно-фторовых октаэдров. При этом, надо осознавать, что нет возможности однозначно сопоставить данную локальную систему с определенным октаэдром, содержащим ион $\mathrm{Cr}^{3+}$, то есть определить какой парамагнитный центр обусловливает данный спектр со связанными с ним главными осями. Тем не менее оказалось, что z-ось локальной системы координат проходит через все октаэдры $\mathrm{CrO}_{4} \mathrm{~F}_{2}$ в структуре $\mathrm{Na}_{5} \mathrm{AlF}_{2}\left(\mathrm{PO}_{4}\right)_{2}$ [2] вблизи центров треугольных граней O-O-F (рис. 4.1).

Этот факт довольно удивителен, учитывая заметное сжатие исходного октаэдра $\mathrm{AlO}_{4} \mathrm{~F}_{2}$ вдоль направления
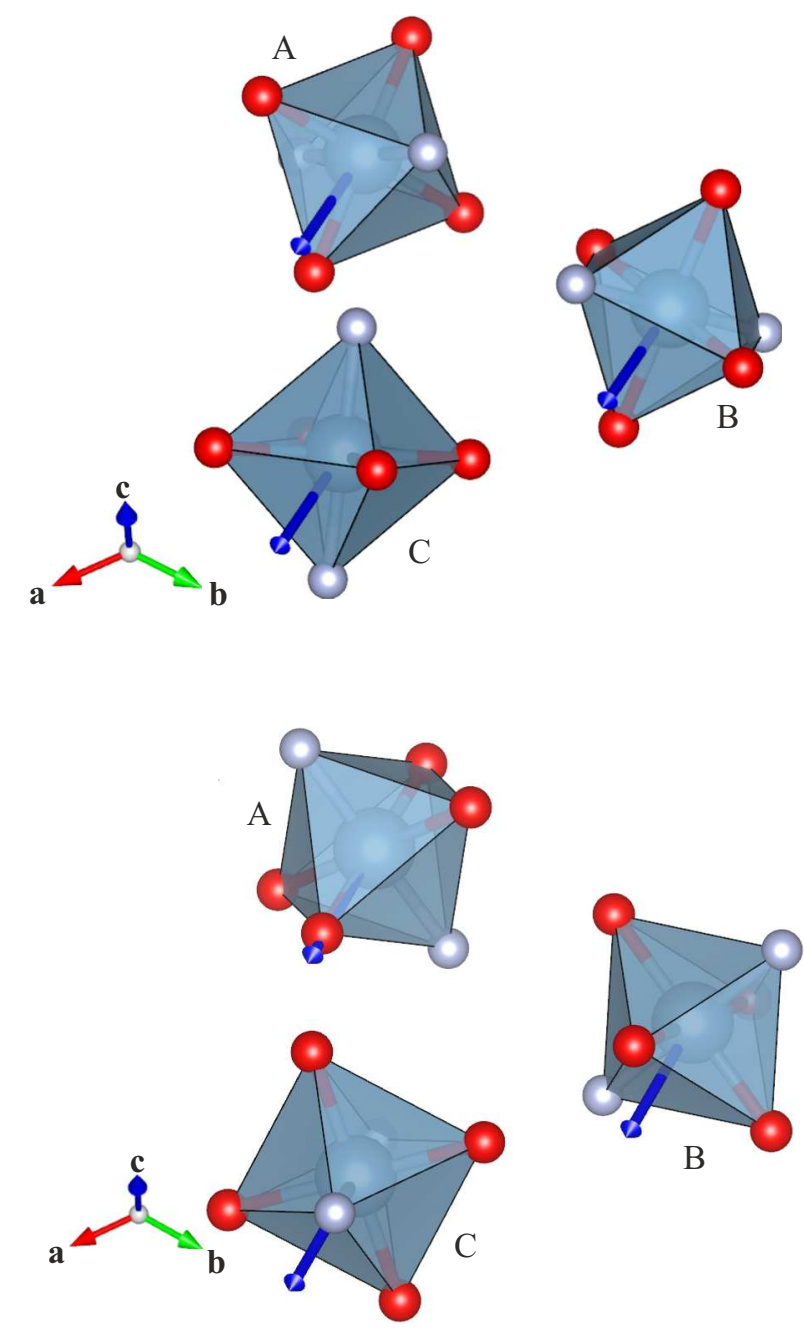

Рис. 4. Ориентация оси $z$ локальной системы координат в структуре трех полиэдров $\mathrm{AlO}_{4} \mathrm{~F}_{2} .1$ - данные о структуре [2], 2 - данные о структуре [3]. abc - кристаллографическая система координат. Красные сферы - кислород, серые фтор. 

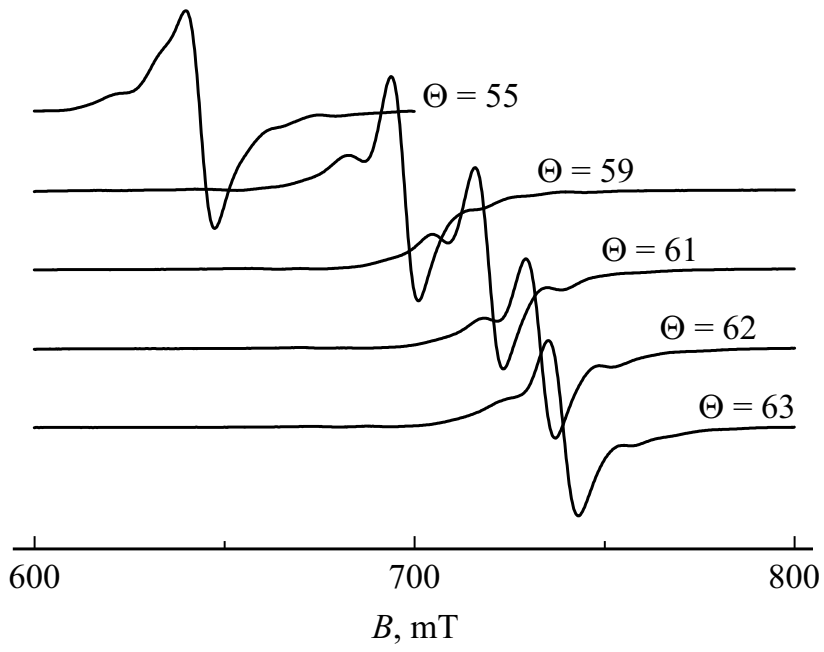

Рис. 5. Вид перехода 1-2 центра $\mathrm{Cr}^{3+}$ с сателлитами при углах $\theta$, соответствующих рис. 2.

фтор-фтор [2,3], то есть вдоль квази-оси четвертого порядка октаэдра. Однако, согласно работам [11-13], только главные оси тензора тонкой структуры четвертого (возможно шестого) ранга отражают направления осей связи искаженных октаэдрических, кубических и тетраэдрических кластеров. Параметры тензора второго ранга и их главные оси нетривиально зависят от характера искажений полиэдра, включая и те, которые возникают из-за замены катиона, и типа взаимодействия с лигандами. Для их расчета необходимо задействовать сложные механизмы, включающие совместное действие кристаллического поля и спин-орбитального взаимодействия, а также учитывать релаксацию окружения примесного иона.

Иная ситуация складывается при наложении оси $z$ локальной системы координат на структуру полиэдров $\mathrm{AlO}_{4} \mathrm{~F}_{2}$ (рис. 4.2), полученную авторами [3]. В двух октаэдрах $\mathrm{AlO}_{4} \mathrm{~F}_{2}$ ось $z$ оказывается близка к их квазитригональным осям, тогда как в октаэдре А (рис. 4.2) эта ось совпадает с диагональю кислородного „квадрата“. Следует заметить, что и на рис. 4.1 локальная ось в октаэдре А несколько повернута от квази-тригональной оси к направлению на ион кислорода. Также можно заметить, что на рис. 4.2 для октаэдра С наблюдается смещение главной оси парамагнитного центра к направлению фтор-фтор. Таким образом, в связи с низким спином исследованного парамагнитного центра остается нерешенным вопрос, которая из работ $[2,3]$ лучше отражает структуру изучаемого кристалла.

При исследовании ориентационного поведения спектра было замечено, что переходы центров $\mathrm{Cr}^{3+}$ сопровождаются несколькими высоко- и низкополевыми сателлитами (см. рис. 5). Такие сателлиты можно объяснить наличием центров $\mathrm{Cr}^{3+}$ в позиции $\mathrm{Al}^{3+}$, ассоциированных с далекими дефектами. Похожая ситуация была обнаружена нами в сегнетоэлектрическом германате свинца, легированном гадолинием и галогенами, где кроме тригональных центров $\mathrm{Gd}^{3+}$ наблюдались триклинные центры $\mathrm{Gd}^{3+}$, ассоциированные с междоузельными ионами галогенов, удаленными от парамагнитного иона на расстояние $>6 \AA[14,15]$.

В кристалле, демонстрирующем ионную проводимость, следует ожидать довольно большую концентрацию вакансий натрия и, следовательно, компенсирующих их заряд вакансий кислорода и фтора. Присутствие в окружении парамагнитного иона далекой вакансии приводит к небольшому изменению параметров спинового гамильтониана и ориентации локальной системы координат, а значит и к небольшому сдвигу положений переходов таких центров.

\section{5. Заключение}

В $X$-диапазоне исследованы спектры парамагнитного резонанса легированных хромом монокристаллов $\mathrm{Na}_{5} \mathrm{AlF}_{2}\left(\mathrm{PO}_{4}\right)_{2}$. Обнаружены интенсивные спектры ионов $\mathrm{Cr}^{3+}$, заместивших ионы $\mathrm{Al}^{3+}$ в триклинных позициях, определены параметры тонкой структуры как в прямоугольной системе $X Y Z$, оси которой связаны с кристаллографическими осями, так и в локальной системе координат. В системе главных осей спектры этих центров описываются спиновым гамильтонианом ромбической симметрии. Кроме того, в этих кристаллах наблюдаются триклинные центры ванадия, ионы $\mathrm{Cr}^{3+}$ в позиции $\mathrm{Al}^{3+}$, ассоциированные с далекими заряженными дефектами (вероятно вакансиями), а также неидентифицированные центры триклинной симметрии.

\section{Благодарности}

Авторы благодарны Б.К. Севастьянову и В.Ф. Тарасову за предоставление образцов, Г.С. Шакурову - за интерес к работе, В.А. Шустову - за ориентацию кристаллов на рентгеновском дифрактометре.

\section{Финансирование работы}

Работа выполнена при финансовой поддержке Министерства науки и высшего образования Российской Федерации, тема № FEUZ-2020-0054.

\section{Конфликт интересов}

Авторы заявляют, что у них нет конфликта интересов.

\section{Список литературы}

[1] Randy Jalem, Ryosuke Natsume, Masanobu Nakayama, Toshihiro Kasuga. J. Phys. Chem. C 120, 1438 (2016).

[2] J. Arlt, M. Jansen, H. Klassen, G. Schimmel, G. Heymer. Z. Anorg. Allg. Chem. 547, 179 (1987).

[3] D.M. Poojary, A. Clearfield, V.A. Timofeeva, S.E. Sigaryov. Solid State Ionics 73, 75 (1994). 
[4] А.К. Иванов-Шиц, С.Е. Сигарёв, В.А. Тимофеева. ФТТ 32, 624 (1990)

[5] A.K. Ivanov-Shits, S.E. Sigaryov. Solid State Ionics 40-41, 76 (1990).

[6] В.А. Тимофеева. Рост кристаллов из растворов-расплавов. Наука, М. (1978). 267 c.

[7] С.А. Альтшулер, Б.М. Козырев. Электронный парамагнитный резонанс соединений элементов промежуточных групп. Наука, М. (1972). C. 121.

[8] T.P.P. Hall, W. Hayes, R.W.H. Stevenson, J. Wilkens. J. Chem. Phys. 38, 1977 (1963).

[9] М.М. Зарипов, В.С.'Кропотов, Л.Д. Ливанова, В.Г. Степанов. ФТТ 9, 209 (1967).

[10] М.М. Зарипов, В.С. Кропотов, Л.Д. Ливанова, В.Г. Степанов. ФТТ 10, 325 (1968).

[11] J.M. Gaite, G.R. Bulka, N.M. Hasanova, N.M. Nisamutdinov, V.M. Vinokurov. J. Phys. C 19, 2077 (1986)

[12] Н.М. Низамутдинов, Н.М. Хасанова, Г.Р. Булка, В.М. Винокуров, И.С. Рез, В.М. Гармаш, Н.И. Павлова. Кристаллография 32, 695 (1987).

[13] В.А. Важенин, М.Ю. Артёмов, А.П. Потапов, В.А. Чернышёв, А.В. Фокин, А.В. Сердцев. ФТТ 59, 942 (2017).

[14] В.А. Важенин, К.М. Стариченко. ФТТ 28, 1916 (1986).

[15] В.А. Важенин, К.М. Стариченко, А.В. Гурьев, Л.И. Левин, Ф.М. Мусалимов. ФТТ 29, 409 (1987).

Редактор Т.Н. Василевская 